\title{
Amyloidosis: an unusual cause of upper gastrointestinal bleeding
}

\author{
Keith Siau, ${ }^{1,2}$ Amera Elzubeir, ${ }^{2}$ Sheldon C Cooper, ${ }^{2}$ Tariq Iqbal ${ }^{3}$
}

${ }^{1}$ Department of Gastroenterology, Royal Wolverhampton Hospitals NHS Trust, Wolverhampton, UK ${ }^{2}$ University Hospital Birmingham NHS Foundation Trust, Birmingham, UK ${ }^{3}$ Department of Gastroenterology, Queen Elizabeth Hospital, Birmingham, UK

\section{Correspondence to}

Dr Keith Siau, keith@siau.org

Accepted 7 October 2016

\section{SUMMARY}

We report a rare case of upper gastrointestinal bleeding in a 55-year-old man with monoclonal gammopathy of unknown significance presenting with abdominal pain, weight loss and melaena. Gastroscopy was unremarkable, but melaena persisted, with the development of symptomatic anaemia. While colonoscopy excluded a lower gastrointestinal aetiology, CT revealed jejunitis, confirmed at capsule endoscopy. Histopathological examination of specimens obtained at single balloon enteroscopy revealed an unusual aetiology: small bowel AL-amyloidosis. We review his clinical presentation, radiological, endoscopic and histological findings and review the literature of this unusual condition.

\section{BACKGROUND}

- We highlight amyloidosis as a potentially fatal condition which can masquerade as monoclonal gammopathy of unknown significance and can result in upper gastrointestinal bleeding (UGIB).

- Awareness of this association is important, as clinicians need to specifically request for Congo Red staining of biopsy specimens to maximise histological sensitivity.

- In addition, we present the first case of amyloid-related iatrogenic perforation.

- We believe that this case report will be of interest to general physicians, as it covers important themes in haematology and gastroenterology.

\section{CASE PRESENTATION}

A 55-year-old Afro-Caribbean man presented to the Emergency Department with a 1 day history of abdominal pain, melaena and postural dizziness. There was unquantified recent weight loss, with no history of fever or diarrhoea. He denied significant medical history and was not taking regular medications. He was an active smoker, with no history of alcohol excess, recent travel or contact with tuberculosis.

On assessment, he was normotensive with sinus tachycardia. He had mild epigastric tenderness without organomegaly or lymphadenopathy. Melaena was confirmed at digital rectal examination. Initial investigation results included haemoglobin $116 \mathrm{~g} / \mathrm{L}$, mean corpuscular volume (MCV) $91.1 \mathrm{fL}$, urea $11.5 \times 10^{9} / \mathrm{L}$, creatinine $64 \mu \mathrm{mol} / \mathrm{L}$ and International Normalised Ratio (INR) 1.3. Liver function tests and bone profile were normal. Gastroscopy and duodenal (D2) biopsies were unremarkable. However, melaena continued with a fall in haemoglobin to $76 \mathrm{~g} / \mathrm{L}$, requiring transfusion support. Computerised tomography enterography (CTE) showed diffuse jejunitis with small mesenteric lymph nodes (figure 1), without radiological evidence of active bleeding.

Following a period of clinical stability and cessation of melaena, the patient was discharged with outpatient capsule endoscopy (CE), colonoscopy and reassessment interval CTE scheduled. It subsequently emerged that he had been diagnosed with monoclonal gammopathy of unknown significance (MGUS) in 2004. Haematology investigations were requested in view of this (figure 2), which were consistent with stable MGUS. Colonoscopy to the caecum was unremarkable, while interval CT abdomen revealed progression of jejunitis and mesenteric lymphadenopathy. CE confirmed jejunitis with mucosal oedema (figure 3). Single balloon enteroscopy, undertaken to obtain biopsy specimens, revealed mucosal congestion and friability within the distal duodenum, limiting insertion to two balloon exchanges. Only two small bowel biopsies were obtained due to mucosal friability. Within 2 hours, the patient developed severe abdominal pain with peritonism. Abdominal CT confirmed a duodenal perforation (figure 4). The patient was managed conservatively with intravenous antibiotics, bowel rest and parenteral nutrition. He made a slow but complete recovery without the need for surgical intervention.

Histological assessment from the small bowel biopsies along with immunohistochemistry identified plasma cells with predominant $\lambda$-light chain expression. Congo red stain was positive with apple green birefringence under polarised light (figure 5), identifying extracellular deposits of amyloid in the mucosa and submucosa, pathognomonic for AL-amyloidosis. Following haematology review, bone marrow trephine biopsy was performed showing $5 \%$ plasma cell infiltration with $\lambda$-chain predominance, staining negative for Congo Red, excluding marrow infiltration. Serial CT imaging confirmed a resolving perforation, and the patient was discharged after a 4-week admission. He was referred to a national amyloid centre where he underwent assessment for systemic involvement. Investigations including serum troponin, echocardiography, urinary protein-creatinine ratio, CT chest and serum amyloid $\mathrm{P}$ (SAP) scintigraphy were normal. He has since been started on empirical therapy with cyclophosphamide, bortezomib and dexamethasone.

\section{DISCUSSION}

Our patient presented with weight loss, recurrent occult UGIB, paraproteinaemia (within MGUS 
Figure 1 ( $A$ and $B)$ CTE with intravenous contrast (axial and coronal views) demonstrating jejunal wall thickening and dilation most notable in the left upper quadrant. Mesenteric nodes were too small for percutaneous biopsy.

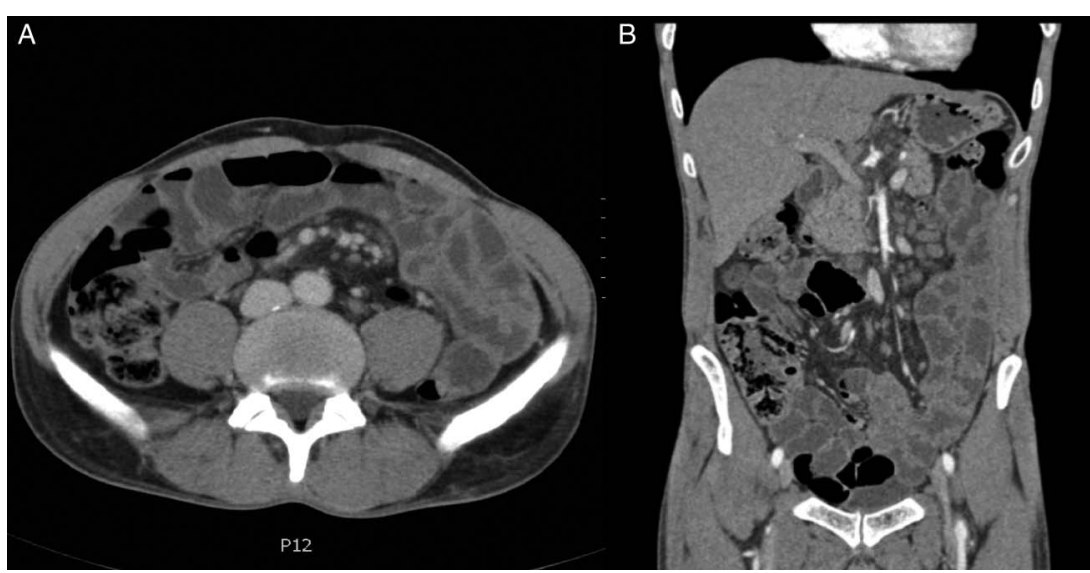

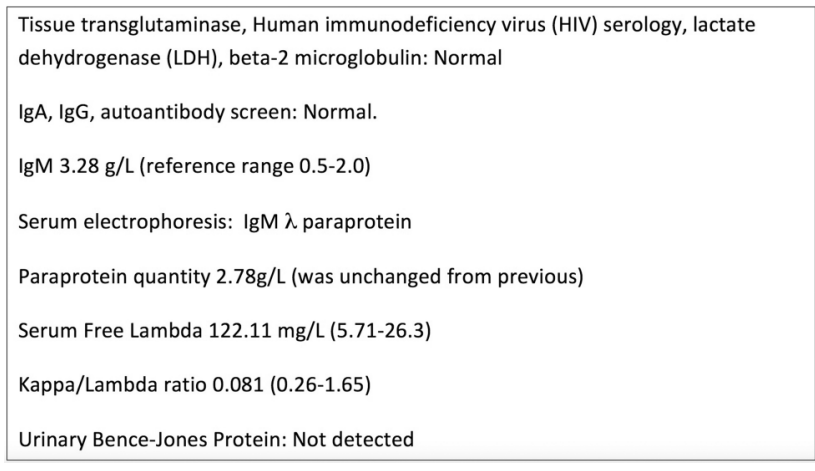

Figure 2 Interim haematology and biochemistry results.

range), jejunitis and mesenteric lymphadenopathy. His differential diagnoses had included lymphoma, small bowel malignancy, atypical infection (including Whipple's disease), Crohn's disease, coeliac disease and autoimmune enteropathy. As such, further endoscopic and histological evaluation was felt to be necessary. Unfortunately, our patient developed a perforation following balloon enteroscopy. The perforation risk with anterograde balloon enteroscopy has been estimated at $0.2 \%{ }^{1}$ Although case reports of spontaneous perforation of the small bowel and colon have been published, we found no reports of perforation after enteroscopy or small bowel biopsy in AL-amyloidosis. Owing to the friable nature of the distal duodenal mucosa, we suspect that the biopsy, which attained submucosal tissue, was sufficient to induce perforation. If amyloidosis had been suspected, a laparoscopic approach may have been considered to be safer.
First described by Virchow in $1854,{ }^{2}$ amyloid (meaning 'starch-like') referred to non-branching fibrils observed microscopically in brain tissue. Nowadays, the term encompasses over 27 heterogenous proteins, ${ }^{3}$ each with their propensity to misfold into $\beta$-pleated sheet configurations, form insoluble extracellular aggregates which stain under Congo Red and infiltrate tissue leading to different patterns of organ dysfunction. Precursor fibrils are derived from monoclonal immunoglobulin light chains in AL (primary) amyloidosis, serum Amyloid A in AA (reactive) amyloidosis and 32 -microglobulin in dialysis-related amyloidosis. AL-amyloidosis is diagnosed on histological confirmation of amyloid and demonstration of a plasma cell dyscrasia (PCD).

PCDs are characterised by abnormal paraprotein production, consisting of MGUS, myeloma, Waldenstrom's macroglobulinaemia (WM) and AL-amyloidosis. ${ }^{4}$ It can be confirmed using serum and urinary protein electrophoresis and immunofixation, serum free light chain (sFLC) estimation or from bone marrow examination. ${ }^{5}$ MGUS, the commonest dyscrasia affecting $3 \%$ over the age of 50 years, ${ }^{6}$ is defined as the presence of paraprotein without significant paraproteinaemia $(<30 \mathrm{~g} / \mathrm{L})$, bone marrow plasma cell excess $(<10 \%)$ or target organ damage, without an alternate PCD. ${ }^{4}$ The potential for transformation from MGUS to myeloma or WM is well recognised and estimated at $1 \% /$ year, ${ }^{6}$ and both are well-recognised precursors of AL-amyloidosis. Data from a Mayo Clinic cohort of MGUS patients indicates that MGUS may be an independent risk factor for developing AL-amyloidosis, with a relative risk of $8.4,{ }^{6}$ and a median lag of 9 years. ${ }^{7}$ The trigger for amyloidogenicity is unclear but arises from abnormal plasma cells developing somatic mutations of the VL6 gene (encoding the variable light chain region), ${ }^{8}$ which alters the isoelectric integrity of synthesised light chains and increases the risk of dissociation into
Figure 3 Capsule enteroscopy images demonstrating duodenal and jejunal mucosal oedema.

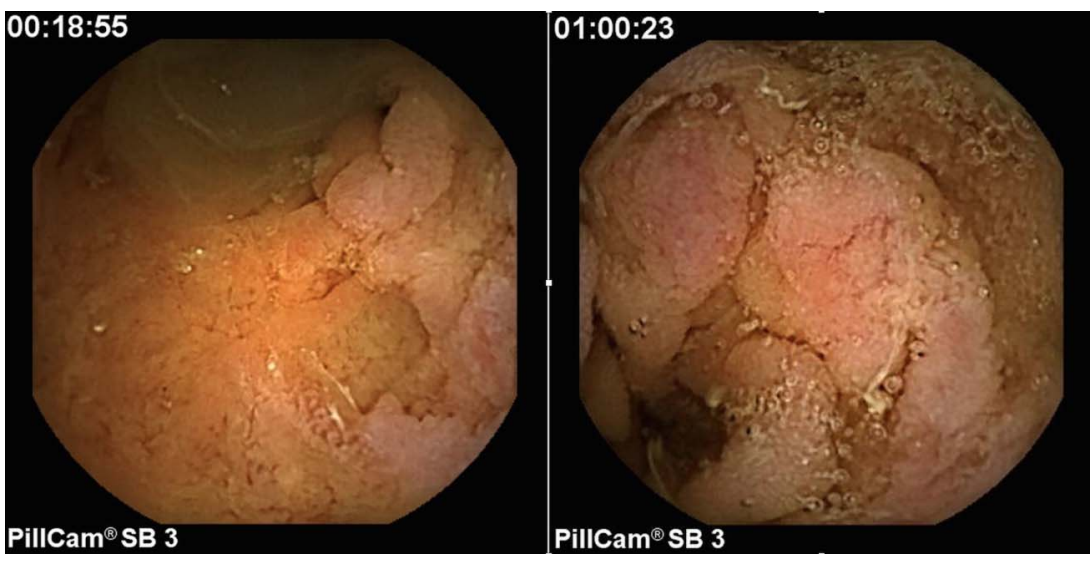

Siau K, et al. BMJ Case Rep 2016. doi:10.1136/bcr-2016-217653 
Figure $4 \mathrm{CT}$ abdomen demonstrating a perforation (yellow circle) at the third part of the duodenum (D3) with retroperitoneal and intraperitoneal free gas.
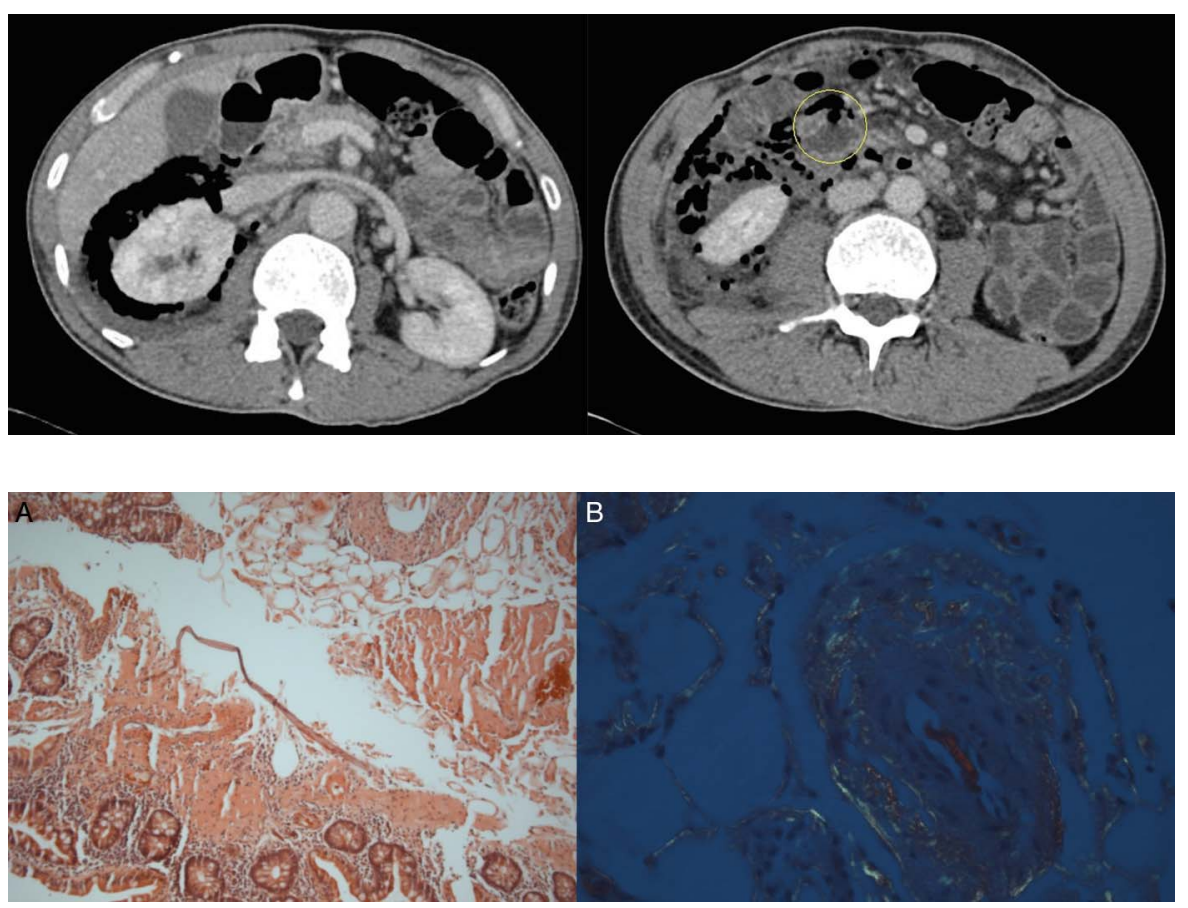

Figure 5 (A) Positive Congo Red staining of duodenal biopsy. (B) Duodenal mucosa with apple green birefringence under polarised light microscopy. insoluble amyloid monomers. 9 The ratio of amyloid formation between $\lambda$-light and $\kappa$-light chains is $3: 1$, suggesting amyloidogenicity inherent to the $\lambda$-chain structure. ${ }^{5}$ Although serum paraprotein levels correlate with risk of transformation to myeloma/WM, ${ }^{6}$ this is not the case for AL-amyloidosis, where fewer than $10 \%$ of patients have a serum paraprotein $>10 \mathrm{~g} / \mathrm{L}$ (after excluding myeloma-related cases). ${ }^{5}$ Interestingly, from a study of 62 patients with MGUS who underwent periumbilical fat tissue aspiration, $14 \%$ stained positive for Congo Red. ${ }^{10}$ In patients with chronic MGUS (median duration of 11 years), 55\% had positive staining. ${ }^{10}$ It is therefore unclear whether such patients have MGUS with transformation to AL-amyloidosis, or AL-amyloidosis masquerading asymptomatically as MGUS.

Although renal, cutaneous and cardiac deposits are common, gastrointestinal (GI) tract involvement occurs in only 3-8\% of AL-amyloidosis ${ }^{11} 12$ and may be complicated by GI dysmotility, obstruction, malabsorption, organomegaly, liver function test derangement, bleeding and rarely, spontaneous bowel perforation. ${ }^{13} 14$ Histologically confirmed deposits are most often detected in the small bowel (50\%), followed by stomach (44\%), colon (32\%), oesophagus (12\%) and rectum (8\%). ${ }^{11}$ Endoscopic findings suggestive of AL-amyloidosis include mucosal friability, submucosal haematoma, polypoid intrusions and thickening of the valvulae conniventes. ${ }^{15} 16$ Histologically, amyloid deposits are found in the muscularis mucosae, submucosa and muscularis propriae. ${ }^{15}$ In contrast, mucosal granularity and lamina propria infiltration is more specific for AA-amyloidosis, which may explain the higher prevalence of diarrhoea and GI bleeding compared to AL-amyloidosis. ${ }^{15}$ UGIB has been reported in up to $36 \%$ of patients with GI amyloidosis. Postulated pathophysiological mechanisms for bleeding include (1) submucosal deposition with necrotic ulceration and mucosal friability, (2) vessel friability/ischaemia due to amyloid angiopathy ${ }^{17}{ }^{18}$ and (3) acquired bleeding diathesis (evident in 51\%), due to Factor X deficiency, hyperfibrinolysis, platelet dysfunction and Vitamin K deficiency. ${ }^{18}$ Acquired Factor X deficiency affects $\sim 14 \%$ of patients ${ }^{17}$ and occurs due to adsorption of Factor $\mathrm{X}$ by amyloid fibrils. ${ }^{18}$ The British Committee for Standards in Haematology $(\mathrm{BCSH})$ have recommended for Factor $\mathrm{X}$ concentrate replacement in such patients, ${ }^{5}$ in addition to haemostatic adjuncts (antifibrinolytic agents and platelet support), which may be of benefit in management of a case of UGIB.

The prognosis of AL-amyloidosis with GI involvement is poor, with a median survival of 6.3 years. ${ }^{11}$ Therapy has traditionally involved myeloma-based treatments, consisting of melphalan, bortezomib, thalidomide and stem cell transplantation. ${ }^{11} 19$ Outcomes are variable, with complete remission seen in $43 \% .^{11}$ Treatment is indicated in systemic disease, coagulopathy and in localised disease with high sFLC levels (due to high risk of progression to systemic disease), ${ }^{20}$ which was the case for our patient. We demonstrate that AL-amyloidosis is a rare cause of UGIB. Endoscopists should be aware of the condition, as $16.7 \%$ of cases are incidentally diagnosed on endoscopy, ${ }^{11}$ and early detection and treatment correlate with improved outcomes. ${ }^{11} 1920$

\section{Learning points}

Amyloidosis should be suspected in patients with unexplained gastrointestinal symptoms, including occult UGIB.

- Monoclonal gammopathy of unknown significance (MGUS) is not a benign condition; AL-amyloidosis may masquerade as MGUS for over 10 years before manifesting with clinical symptoms.

- Congo Red staining should be requested in histological specimens, especially in patients with GI symptoms and coexisting paraproteinaemia/MGUS.

- Balloon enteroscopy with biopsies is helpful for tissue acquisition beyond the reach of conventional gastroscopy but should be performed with caution when amyloidosis is suspected due to high perforation risk.

- Recent guidance for the diagnosis, investigation and management of $\mathrm{AL}$-amyloidosis have been published by the British Committee for Standards in Haematology. 519 
Acknowledgements The authors thank Dr Kassiani Skordilis for providing histology images.

Contributors KS wrote the case presentation and discussion, AE helped with the case presentation, SCC and TI contributed to the discussion and provided guidance for the case report. All authors read and approved the manuscript prior to submission.

Competing interests None declared.

Patient consent Obtained.

Provenance and peer review Not commissioned; externally peer reviewed.

\section{REFERENCES}

1 Gerson LB, Tokar J, Chiorean M. Complications associated with double balloon enteroscopy at nine US centers. Clin Gastroenterol Hepatol 2009;7:1177-82, 1182. e1-3.

2 Sipe JD, Cohen AS. Review: History of the Amyloid Fibril. J Struct Biol 2000;130:88-98.

3 Sipe JD, Benson MD, Buxbaum JN, et al. Amyloid fibril protein nomenclature: 2010 recommendations from the nomenclature committee of the International Society of Amyloidosis. Amyloid 2010;17:101-4.

4 The International Myeloma Working Group. Criteria for the classification of monoclonal gammopathies, multiple myeloma and related disorders: a report of the International Myeloma Working Group. Br J Haematol 2003;121:749-57.

5 Gillmore JD, Wechalekar A, Bird J, et al. Guidelines on the diagnosis and investigation of AL amyloidosis. Br J Haematol 2015;168:207-18.

6 Kyle RA, Therneau TM, Rajkumar SV, et al. A long-term study of prognosis in monoclonal gammopathy of undetermined significance. N Engl J Med 2002:346:564-9.

7 Kyle RA. "Benign" monoclonal gammopathy-after 20 to 35 years of follow-up. Mayo Clin Proc 1993;68:26-36.
8 Abraham RS, Geyer SM, Price-Troska TL, et al. Immunoglobulin light chain variable (V) region genes influence clinical presentation and outcome in light chain-associated (AL) amyloidosis. Blood 2003;101:3801-8.

9 Brumshtein B, Esswein SR, Landau $M$, et al. Formation of amyloid fibers by monomeric light chain variable domains. J Biol Chem 2014;289:27513-25.

10 Miliani A, Bergesio F. AL-amyloidosis in monoclonal gammopathies. Haematologica 1994;79:364-6.

11 Cowan AJ, Skinner M, Seldin DC, et al. Amyloidosis of the gastrointestinal tract: a 13-year, single-center, referral experience. Haematologica 2013;98:141-6.

12 Merlini G, Bellotti V. Molecular mechanisms of amyloidosis. N Engl J Med 2003:349:583-96.

13 Fraser AG, Nicholson GI. Duodenal perforation in primary systemic amyloidosis. Gut 1992;33:997-9.

14 Petre S, Shah IA, Gilani N. Review article: gastrointestinal amyloidosis_clinical features, diagnosis and therapy. Aliment Pharmacol Ther 2008;27:1006-16.

15 Tada S, lida M, Yao T, et al. Endoscopic features in amyloidosis of the small intestine: clinical and morphologic differences between chemical types of amyloid protein. Gastrointest Endosc 1994;40:45-50.

16 Hokama A, Kishimoto K, Nakamoto M, et al Endoscopic and histopathological features of gastrointestinal amyloidosis. World J Gastrointest Endosc 2011:3:157-61.

17 Mumford AD, O'Donnell J, Gillmore JD, et al. Bleeding symptoms and coagulation abnormalities in 337 patients with AL-amyloidosis. $\mathrm{Br} J$ Haematol 2000;110:454-60.

18 Sucker C, Hetzel GR, Grabensee B, et al. Amyloidosis and bleeding: pathophysiology, diagnosis, and therapy. Am J Kidney Dis 2006;47:947-55.

19 Wechalekar $A D$, Gillmore JD, Bird J, et al. Guidelines on the management of $A L$ amyloidosis. Br J Haematol 2015;168:186-206.

20 Dispenzieri A, Lacy MQ, Katzmann JA, et al. Absolute values of immunoglobulin free light chains are prognostic in patients with primary systemic amyloidosis undergoing peripheral blood stem cell transplant. Blood 2006;107:3378-83.

Copyright 2016 BMJ Publishing Group. All rights reserved. For permission to reuse any of this content visit http://group.bmj.com/group/rights-licensing/permissions.

BMJ Case Report Fellows may re-use this article for personal use and teaching without any further permission.

Become a Fellow of BMJ Case Reports today and you can:

- Submit as many cases as you like

- Enjoy fast sympathetic peer review and rapid publication of accepted articles

- Access all the published articles

- Re-use any of the published material for personal use and teaching without further permission

For information on Institutional Fellowships contact consortiasales@bmjgroup.com

Visit casereports.bmj.com for more articles like this and to become a Fellow 